\title{
Cytochrome P450 2D-mediated metabolism is not necessary for tafenoquine and primaquine to eradicate the erythrocytic stages of Plasmodium berghei
}

Erin E. Milner ${ }^{1 *}$, Jonathan Berman ${ }^{1}$, Diana Caridha', Samuel P. Dickson², Mark Hickman ${ }^{1}$, Patricia J. Lee ${ }^{1}$, Sean R. Marcsisin' ${ }^{1}$, Lisa T. Read ${ }^{2}$, Norma Roncal ${ }^{1}$, Brian A. Vesely ${ }^{1}$, Lisa H. Xie' ${ }^{1}$, Jing Zhang ${ }^{1}$, Ping Zhang ${ }^{1}$ and Qigui Li ${ }^{1}$

\begin{abstract}
Background: Due to the ability of the 8-aminoquinolines (8AQs) to kill different stages of the malaria parasite, primaquine $(\mathrm{PQ})$ and tafenoquine $(\mathrm{TQ})$ are vital for causal prophylaxis and the eradication of erythrocytic Plasmodium sp. parasites. Recognizing the potential role of cytochrome (CYP) 450 2D6 in the metabolism and subsequent hepatic efficacy of 8-aminoquinolines, studies were designed to explore whether CYP2D-mediated metabolism was related to the ability of single-dose PQ and TQ to eliminate the asexual and sexual erythrocytic stages of Plasmodium berghei.

Methods: An IV P. berghei sporozoite murine challenge model was utilized to directly compare causal prophylactic and erythrocytic activity (asexual and sexual parasite stages) dose-response relationships in C57BL/6 wild-type (WT) mice and subsequently compare the erythrocytic activity of PQ and TQ in WT and CYP2D knock-out (KO) mice.

Results: Single-dose administration of either $25 \mathrm{mg} / \mathrm{kg}$ TQ or $40 \mathrm{mg} / \mathrm{kg}$ PQ eradicated the erythrocytic stages (asexual and sexual) of P. berghei in C57BL WT and CYP2D KO mice. In WT animals, the apparent elimination of hepatic infections occurs at lower doses of PQ than are required to eliminate erythrocytic infections. In contrast, the minimally effective dose of TQ needed to achieve causal prophylaxis and to eradicate erythrocytic parasites was analogous.
\end{abstract}

Conclusion: The genetic deletion of the CYP2D cluster does not affect the ability of PQ or TQ to eradicate the blood stages (asexual and sexual) of $P$. berghei after single-dose administration.

\section{Background}

Primaquine $(\mathrm{PQ})$ continues to be an indispensable drug for radical cure and presumptive anti-relapse therapy (PART or terminal prophylaxis) of Plasmodium vivax and Plasmodium ovale infections, while serving as an alternative for primary prevention [1,2]. PQ is active against the hepatic stages of all human malaria parasites and the only FDA-approved drug active against the dormant hypnozoite stages of $P$. vivax and $P$. ovale [3]. While there is no clinical benefit to the patient, PQ can be used as a control

\footnotetext{
${ }^{*}$ Correspondence: erin.milner@us.army.mil

1 Walter Reed Army Institute of Research (WRAIR), Silver Spring, MD, USA Full list of author information is available at the end of the article
}

measure to render Plasmodium falciparum sexual blood stages (gametocytes) non-infective to mosquitoes and, therefore, disrupt transmission [4].

Tafenoquine (TQ) is a long half-life PQ analog that eliminates both hepatic and erythrocytic stages of Plasmodium sp. and is currently being developed by the U.S. Army for chemoprophylaxis of all malaria and by GlaxoSmithKline and Medicines for Malaria Venture for the radical cure of $P$. vivax. [5, 6] PQ and TQ are generally well tolerated, but there are known liabilities associated with hemolytic reactions in patients with glucose-6-phosphate dehydrogenase (G6PD) deficiencies [2, 6-10]. Patients should be tested for G6PD deficiency before the multidose regimens of $\mathrm{PQ}$ are prescribed for radical cure, 
terminal prophylaxis, or chemoprophylaxis. The WHO recommends a single administration of $0.25 \mathrm{mg}$ base $/ \mathrm{kg}$ PQ as a relatively well-tolerated (even in G6PD deficient individuals) gametocytocidal dose to inhibit the transmission of $P$. falciparum malaria [4].

In addition to this potential liability leading to the need for G6PD testing prior to dosing, the concept of PQ and TQ as prodrugs in the context of causal prophylaxis and radical cure has introduced another layer of complexity with administration. Recent pre-clinical findings at the Walter Reed Army Institute of Research (WRAIR) have shown that PQ requires CYP2D-mediated metabolism for Plasmodium berghei causal prophylaxis in mice [11, 12]. Tafenoquine was also shown to require CYP2Dmediated metabolism for causal prophylactic activity in $P$. berghei-infected mice [13]. These findings have led some to suggest an 8-aminoquinoline class effect associated with CYP2D-mediated metabolism requirements for activity [14]. Given that nearly ten percent of certain populations are cytochrome P450 (CYP) 2D6 deficient [15], it is clinically important to investigate whether CYP2D-mediated metabolism is related to the ability of PQ and TQ to eradicate the asexual and sexual erythrocytic stages of Plasmodium sp.

To probe this relationship, an established murine $P$. berghei challenge model was utilized involving an intravenous (IV) $P$. berghei sporozoite inoculation on day 0 that results in a hepatic infection followed by subsequent erythrocytic infection on day $3[16,17]$. Vehicle control mice are generally deceased or euthanized due to morbidity within a week. The utility of this model has also been demonstrated for non-8-aminoquinoline structural motifs [16]. As shown in Table 1, administering drugs days $-1,0$, and/or +1 versus day +4 post infection allows for the direct comparison of hepatic causal prophylactic activity versus anti-erythrocytic activity within the same challenge model. The utilization of luciferase-expressing sporozoites from $P$. berghei-infected mosquitoes and an in vivo imaging system (IVIS) permits visualization and quantitation of developing hepatic stages of $P$. berghei for the causal prophylactic assessment of drugs, while flow cytometry (parasitaemia) and microscopy (gametocytaemia) are used to assess erythrocytic infections. To assess the effect of CYP2D-mediated metabolism on the ability of PQ or TQ to eradicate erythrocytic infections, C57BL wild-type (WT) mice were utilized along with knock-out (KO) mice purchased with the murine CYP2D gene cluster deleted and monitored parasitaemia and gametocytaemia [18].

\section{Methods}

\section{Study drugs}

Primaquine (WR002975) and tafenoquine (WR238605) were supplied by the Walter Reed Army Institute of Research (WRAIR) chemical repository. Primaquine (PQ) was supplied as the bisphosphate salt and tafenoquine (TQ) was supplied as the succinate salt. Dosing was calculated based on the milligram per kilogram ( $\mathrm{mg} /$ $\mathrm{kg}$ ) body weight with respect to the molecular weight of the free base. The bulk drug of TQ used for the study was synthesized for the WRAIR by Ash Stevens, Inc. (Detroit, MI USA) with a purity of $>98 \%$. PQ was purchased from Sigma Aldrich (St. Louis, MO USA) with a purity of $>98 \%$.

\section{Sporozoites and viability}

Luciferase-expressing P. berghei (strain ANKA) sporozoites [19] were obtained from laboratory-reared female Anopheles stephensi mosquitoes from the Department of Entomology, Walter Reed Army Institute of Research. The mosquitoes were maintained at $18^{\circ} \mathrm{C}$ for $17-22$ days after feeding on $P$. berghei malaria infected Swiss ICR mice. As the salivary glands were extracted from the malaria-infected mosquitoes, they were stored on ice in RPMI medium with $1 \%$ mouse serum. Sporozoites were recovered by the method of Ozaki [20]. To ensure the inoculated sporozoites were viable following the isolation procedure, they were stained with a vital dye containing fluorescein diacetate $[50 \mathrm{mg} / \mathrm{mL}$ acetone; Sigma Aldrich (St. Louis, MO USA)] and ethidium bromide [ $20 \mathrm{ug} / \mathrm{mL}$ in phosphatebuffered saline; Sigma Aldrich (St. Louis, MO)] and counted in a haemocytometer. The viability of sporozoites ranged from 87 to $100 \%$.

Table 1 In vivo P. berghei intravenous (IV) sporozoite challenge causal prophylactic and erythrocytic models

\begin{tabular}{lll}
\hline Model & Causal prophylactic model & Erythrocytic treatment model \\
\hline Drug administration (day) $^{\mathrm{a}}$ & $-1,0$, and/or +1 & +4 \\
Endpoint $^{\text {b }}$ & Hepatic and erythrocytic parasites (asexual parasitemia) & $\begin{array}{c}\text { Erythrocytic parasites: parasitemia (asexual), gametocytemia } \\
\text { (sexual) }\end{array}$ \\
Technique & IVIS (liver), flow cytometry (parasitemia) & Flow cytometry (parasitemia) microscopy (gametocytemia) \\
\hline
\end{tabular}

a Day of drug administration relative to IV sporozoite inoculation (day 0). IVIS In vivo imaging system

b Animals are monitored for 30 days or recrudescence and subsequent euthanasia due to comorbidities 


\section{Animal housing}

Male 8-12 week-old C57BL CYP2D KO mice, bred using the method Scheer and colleagues [18], were purchased from Taconic (Hudson, NY USA). Male 6-8 week-old C57BL/6 wild-type mice were purchased from Charles River Laboratories (shipped from Raleigh, NC USA). Upon arrival, all animals were acclimated for 7 days in quarantine. The animals were housed in a cage contained in a room with a temperature range of $64-79{ }^{\circ} \mathrm{F}, 34-68 \%$ relative humidity, and a $12 \mathrm{~h}$ light/dark cycle. Food and water were provided ad libitum during quarantine and throughout the study. The animals were fed a standard rodent maintenance diet. The $\mathrm{C} 57 \mathrm{BL} / 6 \mathrm{WT}$ and $\mathrm{C} 57 \mathrm{BL}$ CYP2D KO mice were cautiously housed separately and identified with the appropriate cage cards. Extra care was taken to ensure there were no labelling errors associated with WT and KO mice.

\section{Intravenous sporozoite inoculation}

Sporozoites were rapidly isolated from the same batch of mosquitoes for a given experiment and administered to C57BL/6 wild-type (WT) and CYP2D knock-out (KO) mice the same morning to control for biological and time-dependent variability in sporozoite preparations. Each mouse was inoculated intravenously in the tail vein with 10,000 sporozoites suspended in $0.1 \mathrm{~mL}$ volume on day 0 . The WT group was randomized separately from the $\mathrm{KO}$ group after sporozoite inoculation and prior to dosing with the test drugs or vehicle control. All animal studies were performed under IACUC-approved protocols. All animal experiments were conducted in a facility accredited by the Association for the Assessment and Accreditation of Laboratory Animal Care, International, and in compliance with the Animal Welfare Act and other federal statutes and regulations relating to animals and experiments involving animals and adhere to the principles stated in the Guide for the Care and Use of Laboratory Animals (National Academy Press, 1996).

\section{Genetic verification}

Ear punch pathological samples were collected from a subset of WT and $\mathrm{KO}$ mice and stored in 70\% ethanol at $-80{ }^{\circ} \mathrm{C}$ for $12 \mathrm{~h}$ in preparation for shipment the following day. The samples were blinded with identification numbers and shipped with dry-ice overnight to Taconic, where the biopsies were transferred to a 96-well box and the DNA was extracted using the Qiagen Blood and Tissue extraction $\mathrm{Kit}^{\circledR}$ (Hilden, Germany). After extraction, the DNA was evaluated using a spectrophotometer to determine the concentration and normalized to $10 \mathrm{ng} /$ $\mu \mathrm{L}$. A master mix was prepared using reagents obtained through Qiagen, dNTPs, and the four primers from MWG Operon. The master mix was distributed into the
PCR plate, and upon thermal cycling, the product was processed with a capillary electrophoresis instrument. Reagent, primer information, and assay data are available in the Additional file 1: Figures S1-S4.

\section{Test agent administration}

Drug substances were administered orally on days $-1,0$, and/or +1 (causal prophylactic experiments) or on day 4 (blood-stage experiments) with respect to sporozoite inoculation on day 0. Dosing calculations were based upon body weight measurements obtained on the day of dosing prior to preparing fresh stock solutions of the test compounds by grinding the needed quantity of drug in cold $\left(4{ }^{\circ} \mathrm{C}\right) 0.5 \%(\mathrm{w} / \mathrm{v})$ hydroxyethyl cellulose and $0.2 \%$ $(\mathrm{v} / \mathrm{v})$ Tween-80 (0.5\% HECT) and diluting to the appropriate concentration. Drugs were ground using a ProScientific 300D homogenizer (Oxford, CT) and the particle size was measured using a Horiba LA-950V2 particle size analyzer (West Chicago, IL). Oral doses were delivered via an intragastric feeder (18-gauge) to the designated recipient.

\section{In vivo imaging system (IVIS)}

In vivo imaging studies of bioluminescence activity from luciferase expressing $P$. berghei infected mice were performed using a Perkin Elmer IVIS Spectrum (Hanover, $\mathrm{MD})$. Mice were evaluated at $24 \mathrm{~h}$ post sporozoite inoculation to confirm a liver-stage malaria infection. Mice received $200 \mathrm{mg} / \mathrm{kg}$ luciferin (Gold Biotechnology, St. Louis, MO) intraperitoneally in a volume not to exceed $200 \mu \mathrm{L}$. Five minutes post luciferin administration, the mice were anesthetized in a sleeping chamber with inhaled isoflurane. The mice were then positioned ventral side-up on the IVIS on a $37{ }^{\circ} \mathrm{C}$ platform. The mice continue to receive isoflurane through nose cone delivery. A camera exposure time of $5 \mathrm{~min}$ was utilized for the 24, 48 , and $72 \mathrm{~h}$ time points with f-stop $=1$ and large binning setting. Quantitative analysis of bioluminescence emitted from whole bodies or region of intensity (ROI) were determined by measuring the luminescence signal intensity in photons/second using the ROI settings of the Living Image ${ }^{\circledR} 4.0$ software. The ROI, which measurements were expressed in total flux of photons, was set to measure the bioluminescence signal emitted in the abdominal area at the location of the liver ( 24 and $48 \mathrm{~h}$ ) and/or whole body imaging at $72 \mathrm{~h}$.

\section{Flow cytometry (FCM)}

Mice were analysed for asexual blood stage infections by determination of parasitaemia of tail blood samples $(3 \mu \mathrm{L}$ each) using a FC500 MPL flow cytometer (BeckmanCoulter Co., CA USA), which conducts five-color analysis from either single or dual laser excitation. Infected 
erythrocytes, uninfected erythrocytes, and leukocytes were gated on logarithmic forward/side dot plots. Cells were analysed at an average rate of 2000-3000 erythrocytes/s. Filters were placed before the green (FL1) and red (FL-2) photomultiplier tubes (PMTs) such that the green PMT registered fluorescence emission between 520 and $555 \mathrm{~nm}$, and the red PMT measured emission greater than $580 \mathrm{~nm} .2-3 \mu \mathrm{L}$ of blood from the mouse tail was collected directly into $0.3 \mathrm{~mL}$ of $1 \%$ heparinized isotonic buffer (PBS saline). In this study, $1 \mathrm{~mL} 0.04 \%$ of glutaraldehyde was used for fixation and the samples were then incubated at $4{ }^{\circ} \mathrm{C}$ for $60 \mathrm{~min}$. The cells were centrifuged at $450 \times g$ for $5 \mathrm{~min}$. The supernatant was removed by aspiration and the cells were re-suspended in $0.5 \mathrm{~mL}$ PBS buffer supplemented with $0.25 \%(\mathrm{v} / \mathrm{v})$ Triton X-100 for $10 \mathrm{~min}$ incubation at room temperature. After centrifugation, the permeabilized cells were re-suspended in $0.5 \mathrm{~mL}$ of RNase at $1 \mathrm{mg} / \mathrm{mL}$ concentrations and incubated for at least $2 \mathrm{~h}$ at $37{ }^{\circ} \mathrm{C}$ to ensure complete digestion of reticulocytes. Plasmodium berghei infection in mice results in anaemia and subsequent reticulocytosis. Therefore, high RNAse concentrations for digesting large amounts of reticulocytes RNA were required for assessment of parasitaemia in this mouse model. YOYO-1 dye (from $1 \mathrm{mM}$ stock solution in DMSO) was diluted to $2500 \mathrm{ng} / \mathrm{mL}$ (100-fold) concentrations in PBS and $20 \mu \mathrm{L}$ of YOYO-1 solution at $2500 \mathrm{ng} / \mathrm{mL}$ was added to $0.5 \mathrm{~mL}$ of sample to a final dye concentration of $100 \mathrm{ng} / \mathrm{mL}$ of YOYO-1, which has been shown to be optimal to discriminate infected erythrocytes from the lowest $(0.01 \%)$ to the highest parasitaemia counts $(74.0 \%)[21,22]$. It should be noted that tail blood flow cytometry measurements were performed on 10 naïve mice which all resulted in a background flow cytometry value of $0.3 \%$ parasitaemia. This background value was subtracted from the flow cytometry values obtained for each animal during the study. All values in this manuscript reflect corrected flow parasitaemia with $0.3 \%$ background subtracted.

\section{Slide preparation and light microscopy}

With the mouse restrained, a needle was used to prick the tail vein and one drop of blood was massaged from the vein and directly placed on a $25 \times 75 \mathrm{~mm}$ microscopy slide (Fischer Scientific, USA) labelled with the date and animal ID number. A clean slide was used to "smear" the blood to create a single layer of erythrocytes/ reticulocytes with a "feathered-edge." The thin smear was allowed to dry at ambient room temperature for $4 \mathrm{~h}$ prior to fixing the slides with methanol. $20 \%$ Giemsa (Sigma Aldrich, USA) in phosphate buffer [10L batch: $7 \mathrm{~g}$ potassium phosphate monobasic (Fischer Scientific, USA), 10 g sodium phosphate dibasic (Fischer Scientific, USA), 10L DI water] was utilized to stain the slides for
30 min prior to rinsing with DI water. The stained slides were allowed to dry $12 \mathrm{~h}$ in ambient temperature prior to adhering cover slips (Fischer Scientific, USA) using Poly-Mount ${ }^{\circledR}$ (Polysciences Inc., Warrington, PA). The slides were randomly distributed to 4 microscopists who were provided guidelines for slide reading methodology. Using a $100 \times$ high-power microscope, an eyepiece graticule with a St Andrew's cross, and a layer of immersion oil (Sigma Aldrich, USA), parasitized cells in ten fields were quantified to provide the mean cell percentages associated with the sexual and asexual stages of P. berghei [23-25]. Percent parasitaemia was calculated as $[$ (mean number asexual parasites)/(mean number of total RBCs in one quadrant $\times 4)] \times 100$. The percent gametocytaemia was calculated as [ (mean number mature gametocytes)/(mean number of total RBCs in one quadrant $\times 4)] \times 100$.

\section{Parasitemia correlation}

The quantification of erythrocytic parasites was accomplished using flow cytometry (asexual stages) and microscopy (sexual and asexual parasites). The utilization of flow cytometry allowed for the rapid determination of parasitaemia (asexual parasites) and the results are reflected in the tables and figures of the manuscript. Due to the meticulous nature of the microscopists, both sexual and asexual parasites were quantified. The percent parasitaemia derived from microscopy and flow cytometry was subsequently utilized to correlate the two methods. The relationship between percent parasitaemia determined using flow cytometry versus light microscopy was performed using $\mathrm{R}$ version 3.2.4. Linear and non-linear relationships were analysed utilizing linear and polynomial regression as well as a repeated measures analysis with a spatial power law covariance structure to account for correlation between time points. The correlation serves to link the parasite quantification derived from flow cytometry and microscopy. Correlation results are presented in the Additional file 1: Figure S7.

\section{Pharmacokinetic parameter determination}

Pharmacokinetic (PK) parameters for PQ and TQ in plasma and liver generated by the WRAIR [26, 27] were analysed using noncompartmental analysis (NCA) via the Phoenix-Win-Nonlin software package (version 6.4; Pharsight Corp., Mountain View, CA). The PK parameters were utilized to explore the variability between wildtype and CYP2D knock-out mice.

\section{Statistical analysis}

All tests of significance between groups across days were performed based on a linear regression model with repeated measures fit using a spatial power law 
covariance matrix (similar to the AR(1) model but allowing for unequally spaced days). All models included main effects for treatment group and study day, as well as a group $\times$ day interaction. When comparing mouse strains, the model also included a main effect for strain, the twoway interactions with group and day, and the three-way group $\times$ day $\times$ strain interaction. Significance was based on the means from the interaction terms within day, and correction for multiple testing was based on Tukey's post hoc correction for all pairwise tests. Correlation was analysed based on a simple linear regression model, polynomial regression models up to third-degree polynomials, and a repeated measures model. A LOESS curve was also fit for comparison.

\section{Graphical representations}

Data analysis and graphical representations were generated using either Phoenix-Win-Nonlin software package (version 6.4; Pharsight Corp., Mountain View, CA), Microsoft Excel, and R version 3.2.4.

\section{Results \\ C57BL/6 wild-type (WT) murine causal prophylactic and treatment dose-ranging studies}

Single-dose primaquine (PQ) and tafenoquine (TQ) dose-ranging studies were completed in order to assess the dose-response relationships between causal prophylactic (hepatic) activity and treatment (erythrocytic) activity and establish the dosing regimen for the CYP2D KO versus WT study erythrocytic study.

\section{Primaquine causal prophylactic (hepatic) activity}

PQ was administered for causal prophylaxis on either day 0 and/or day +1 relative to IV sporozoite inoculation on day 0 . Traditionally, the WRAIR administers drugs on days $-1,0,+1$ to assess causal prophylactic activity in the IV sporozoite challenge model $[16,17]$. Given the short half-life and rapid clearance of PQ in C57BL wild-type mice (plasma $\mathrm{t}_{1 / 2}=1.5 \pm 0.4 \mathrm{~h}$; liver

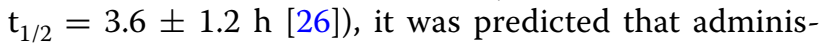
tering PQ on day -1 would not result in the clearance of hepatic parasites on day 0 and was not necessary for causal prophylaxis. This was confirmed when $25 \mathrm{mg} /$ $\mathrm{kg}$ of PQ was administered day 0 and day +1 leading to $100 \%$ suppression of the hepatic IVIS signal and no detected erythrocytic infection after 30 days post infection. Subsequently single-dose PQ $(40,20$, or $10 \mathrm{mg} /$ $\mathrm{kg}$ ) was administered day 0 relative to IV sporozoite inoculation. The 40 and $20 \mathrm{mg} / \mathrm{kg}$ cohorts led to $100 \%$ suppression of the hepatic IVIS signal and no detected erythrocytic infection. The $10 \mathrm{mg} / \mathrm{kg}$ cohort led to $100 \%$ suppression of the hepatic IVIS signal and no erythrocytic infection was detected in 3/5 mice. This suggests that hepatic parasites below the IVIS limit of detection exited the liver to infect the blood. The administration of $40 \mathrm{mg} / \mathrm{kg}$ of PQ day +1 led to incomplete suppression of the hepatic IVIS signal and 0/5 animals free of parasitaemia (Table 2).

\section{Primaquine erythrocytic treatment activity}

Single-dose PQ (10, 20, and $40 \mathrm{mg} / \mathrm{kg}$ ) was administered on day +4 relative to IV sporozoite inoculation and blood samples were analysed using flow cytometry and light microscopy to assess parasitaemia and gametocytaemia, respectively. PQ administration in each cohort led to the initial eradication of erythrocytic parasites followed by recrudescence observed on day 11 . These suggest PQ eradicates hepatic infections at lower doses than are required to eliminate erythrocytic infections (Table 2).

\section{Tafenoquine causal prophylactic (hepatic) activity and erythrocytic treatment activity}

TQ exhibited comparable dose-response relationships for hepatic and erythrocytic reservoirs of parasitaemia in wild-type animals. As shown in Table 3, $20 \mathrm{mg} /$ $\mathrm{kg}$ TQ led to $100 \%$ suppression of the IVIS signal and no detectable erythrocytic infection for $5 / 5$ mice when administered on days $-1,0$, and/or +1 , while lower doses led to either incomplete suppression of the IVIS signal or failure to eradicate the erythrocytic infections within the dosing cohorts. Given the long half-life of $\mathrm{TQ}$ in wild-type mice (plasma $\mathrm{t}_{1 / 2}=53.8 \pm 3.5 \mathrm{~h}$; liver $\mathrm{t}_{1 / 2}=83.5 \pm 2.3 \mathrm{~h}$ [27]), the causal activity of TQ administered on day -1 is likely related to drug remaining in the liver when sporozoites are inoculated on day 0 ; the anti-erythrocytic activity in the absence of causal activity of TQ administered on day +1 could be related to drug remaining in the circulation when parasites emerge from the liver on day +3 . The administration of $20 \mathrm{mpk}$ day +4 led to the eradication of erythrocytic parasites in $4 / 5$ mice with recrudescence observed in $1 / 5$ mice after 29 days (Table 3 ).

\section{Comparison of $\mathrm{PQ}$ or TQ in wild-type (WT) and CYP2D knock-out (KO) C57BL mice}

The disease progression of $P$. berghei in both liver and blood was shown to be analogous in C57BL wild-type (WT) and CYP2D knock-out (KO) mice (refer to Additional file 1: Figure S3). Following IV sporozoite inoculation (day 0), the presence of a liver stage infection was detected at 24 and $48 \mathrm{~h}$ followed by an erythrocytic infection at $72 \mathrm{~h}$ in both WT and CYP2D KO mice. The erythrocytic infection progressed analogously in WT and CYP2D KO vehicle control mice until mortality or euthanasia due to morbidity occurred within approximately one week following infection. 
Table 2 Primaquine (PQ) wild-type (WT) dose-ranging studies using C57BL/6 mice

\begin{tabular}{|c|c|c|c|c|}
\hline Day dose administered ${ }^{a}$ & $P Q$ dose $(\mathrm{mg} / \mathrm{kg})$ & No hepatic IVIS signal ${ }^{b}$ & $\begin{array}{l}\text { No asexual erythrocytic } \\
\text { infection (parasitemia) }^{c}\end{array}$ & $\begin{array}{l}\text { No sexual erythrocytic } \\
\text { infection (gametocytemia) }\end{array}$ \\
\hline \multicolumn{5}{|l|}{ Causal prophylactic model } \\
\hline D0, D1 & $25 \times 2 d$ & $5 / 5(\mathrm{D} 1), 5 / 5(\mathrm{D} 2)$ & $5 / 5$ & NA \\
\hline \multirow[t]{3}{*}{ D0 } & 10 & $5 / 5(\mathrm{D} 1), 5 / 5(\mathrm{D} 2)$ & $3 / 5$ & NA \\
\hline & 20 & $5 / 5(\mathrm{D} 1), 5 / 5(\mathrm{D} 2)$ & $5 / 5$ & NA \\
\hline & 40 & $5 / 5$ (D1), 5/5 (D2) & $5 / 5$ & NA \\
\hline D1 & 40 & $1 / 5(\mathrm{D} 1), 1 / 5(\mathrm{D} 2)$ & $0 / 5$ & NA \\
\hline \multicolumn{5}{|c|}{ Erythrocytic treatment model } \\
\hline \multirow[t]{3}{*}{ D4 } & 10 & NA & $0 / 5$ & $0 / 5$ \\
\hline & 20 & NA & $0 / 5$ & $0 / 5$ \\
\hline & 40 & NA & $0 / 5$ & $0 / 5$ \\
\hline
\end{tabular}

$P Q$ primaquine, $\mathrm{mg} / \mathrm{kg}$ milligrams free base of drug per kg body weight, $N A$ not applicable, IVIS in vivo imaging system

${ }^{a}$ Day of drug administration relative to intravenous sporozoite (IV SPZ) challenge (day 0) using C57BL/6 wild-type mice

${ }^{\mathrm{b}}$ Number of animals without an IVIS signal (indicates no hepatic infection). D1 day 1 (relative to IV SPZ challenge day 0). D2 day 2 (relative to IV SPZ challenge day 0)

c Number of animals without parasitemia (flow cytometry) or gametocytemia (microscopy) day 29. $\mathrm{N}=5$ animals per cohort

Table 3 Tafenoquine (TQ) wild-type (WT) dose-ranging studies using C57BL/6 mice

\begin{tabular}{|c|c|c|c|c|}
\hline Day dose administered $^{a}$ & $P Q$ dose $(\mathrm{mg} / \mathrm{kg})$ & No hepatic IVIS signal $^{b}$ & $\begin{array}{l}\text { No asexual erythrocytic } \\
\text { infection (parasitemia) }\end{array}$ & $\begin{array}{l}\text { No sexual erythrocytic } \\
\text { infection (gametocytemia) }\end{array}$ \\
\hline \multicolumn{5}{|l|}{ Causal prophylactic model } \\
\hline \multirow[t]{4}{*}{ D1 } & 2.5 & 0/5 (D1), 0/5 (D2) & $0 / 5$ & NA \\
\hline & 5 & $3 / 5$ (D1), 2/5 (D2) & $0 / 5$ & NA \\
\hline & 10 & $5 / 5(\mathrm{D} 1), 5 / 5(\mathrm{D} 2)$ & $4 / 5$ & NA \\
\hline & 20 & $5 / 5(\mathrm{D} 1), 5 / 5(\mathrm{D} 2)$ & $5 / 5$ & NA \\
\hline \multirow[t]{4}{*}{ D0 } & 2.5 & 0/5 (D1), 0/5 (D2) & $0 / 5$ & NA \\
\hline & 5 & $4 / 5$ (D1), 4/5 (D2) & $3 / 5$ & NA \\
\hline & 10 & $3 / 5$ (D1), 4/5 (D2) & $2 / 5$ & NA \\
\hline & 20 & $5 / 5(\mathrm{D} 1), 5 / 5(\mathrm{D} 2)$ & $5 / 5$ & NA \\
\hline D1 & 20 & 0/5 (D1), 0/5 (D2) & $5 / 5$ & NA \\
\hline \multicolumn{5}{|l|}{ Erythrocytic treatment model } \\
\hline \multirow[t]{3}{*}{ D4 } & 5 & NA & $0 / 5$ & $0 / 5$ \\
\hline & 10 & NA & $0 / 5$ & $0 / 5$ \\
\hline & 20 & NA & $4 / 5$ & $4 / 5$ \\
\hline
\end{tabular}

TQ tafenoquine, $\mathrm{mg} / \mathrm{kg}$ milligrams free base of drug per kg body weight, NA not applicable, IVIS in vivo imaging system

a Day of drug administration relative to intravenous sporozoite (IV SPZ) challenge (day 0) using C57BL/6 wild-type mice

b Number of animals without an IVIS signal (indicates no hepatic infection). D1 = day 1 (relative to IV SPZ challenge day 0 ). D2 = day 2 (relative to IV SPZ challenge day 0 )

c Number of animals without parasitemia (flow cytometry) or gametocytemia (microscopy) day 29. $\mathrm{N}=5$ animals per cohort

A single dose of drug was administered day +4 post infection to study the effect of CYP 2D-mediated metabolism relative to the ability of PQ and TQ to initially eradicate $P$. berghei erythrocytic stages (rather than determine the optimal dosing regimen to avoid recrudescence). Blood samples were analysed using flow cytometry (asexual stages) and light microscopy (sexual stages) to assess parasitaemia (Additional file 1: Figure S5) and gametocytaemia (mature gametocytes; Additional file 1: Figure 56$)$, respectfully.

Single-dose PQ $(40 \mathrm{mg} / \mathrm{kg})$ and TQ $(25 \mathrm{mg} / \mathrm{kg})$ eradicated erythrocytic parasites in both WT and CYP2D $\mathrm{KO}$ mice for one week post-drug administration. Recrudescence was observed day 11 in the PQ-treated WT and CYP2D KO cohorts (Additional file 1: Figures S5 and S6), which corresponds to the short half-life 
$\left(\right.$ WT t $_{1 / 2}=1.5 \pm 0.4 \mathrm{~h}$; CYP2D KO t $\mathrm{t}_{1 / 2} 3.5 \pm 0.9 \mathrm{~h}$ ) and rapid clearance of $\mathrm{PQ}$ in plasma [26]. Comparing CYP 2D KO versus WT cohorts, no statistically significant differences in mean percent parasitaemia (Fig. 1) were observed for PQ until day 15 , which resolved by day 25 just prior to euthanasia. In regards to gametocytaemia, differences between PQ-treated WT and KO cohorts were not statistically significant except for day 11 (Fig. 2).

For 25 days following single-dose TQ administration, there was no statistical difference between WT and KO cohort mean parasitaemia (Fig. 1). There was no statistically significant difference relative to mean gametocytaemia (Fig. 2) between WT and KO cohorts treated with TQ for the duration of the study. The prolonged duration prior to recrudescence for the TQ-treated animals is likely related to the longer half-life in plasma (WT $\mathrm{t}_{1 / 2}=53.8 \pm 3.5 \mathrm{~h} ;$ CYP2D KO t $\mathrm{t}_{1 / 2}=72.4 \pm 15.5 \mathrm{~h}$ ) and delayed clearance relative to PQ [27].

\section{Pharmacokinetic-pharmacodynamic (PK/PD) analysis}

Figure 3 graphically annotates the plasma and liver fold-changes associated with $20 \mathrm{mg} / \mathrm{kg}$ PQ (Fig. 3a) and $20 \mathrm{mg} / \mathrm{kg}$ TQ (Fig. 3b) CYP2D KO PK parameters versus reference (WT) strain parameters. A value of one represents no relative change in the PK parameter between the $\mathrm{KO}$ and WT mice (logarithmic $\mathrm{x}$-axis).

Two items are noteworthy in regards to the foldchanges representing the PK parameters of the parent compound: (1) In contrast to PQ, the mean PK parameters for TQ generally reside toward the central line, which

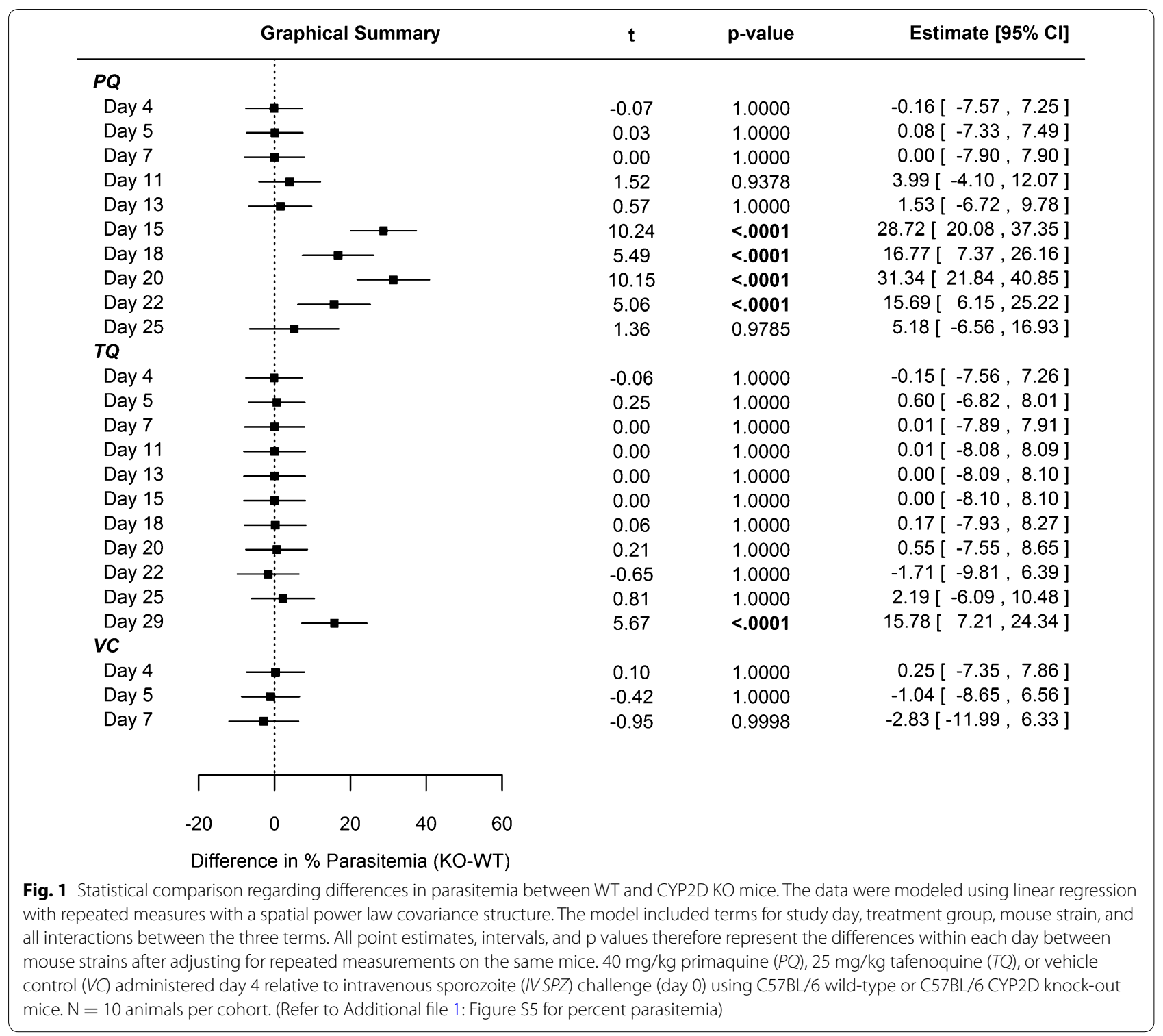




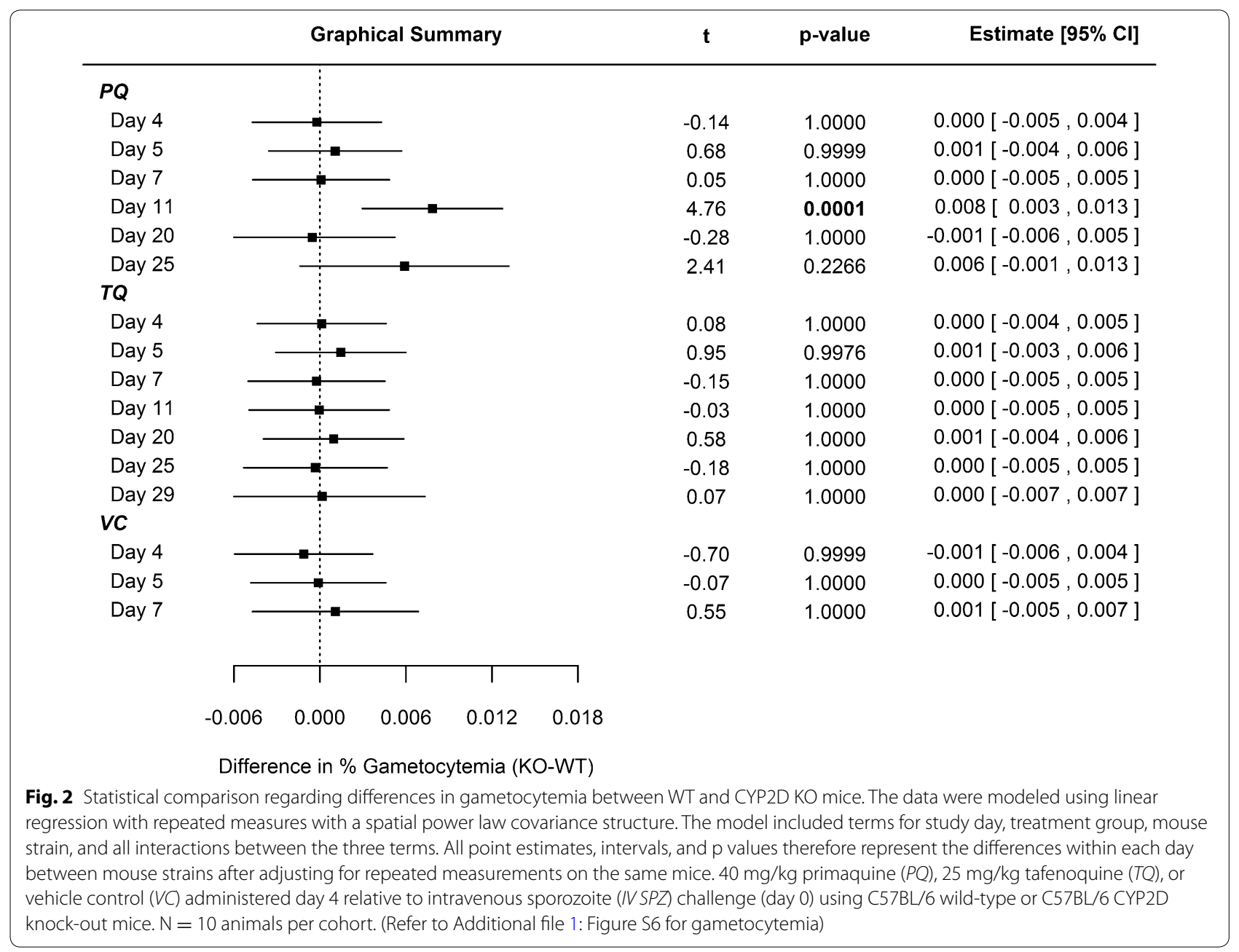

indicates limited relative changes between CYP2D KO and WT mice treated with TQ and (2) both PQ and TQ single-dose administration eradicated erythrocytic parasites despite reduced clearance and increased exposure (AUC) of the parent compound in CYP2D KO mice compared to WT mice. It is important to note that PQ parent exposure is greatly increased in CYP2D $\mathrm{KO}$ vs WT mice, whereas TQ exposure is more modestly increased in CYP2D KO vs WT mice.

\section{Discussion}

Three key observations should be highlighted: (1) PQ eradicates hepatic parasites at lower doses than are required to eradicate erythrocytic parasites, while TQ exhibits an analogous dose-response relationship when comparing hepatic and erythrocytic activity, (2) there was no significant difference between WT and CYP2D KO mice regarding the preliminary eradication of erythrocytic parasites (asexual and sexual) following single-dose administration of both TQ $(25 \mathrm{mg} / \mathrm{kg})$ and PQ (40 mg/ $/ \mathrm{kg})$, and (3) the variability between $\mathrm{WT}$ and CYP2D KO murine PK parameters did not significantly affect the pharmacodynamic endpoints of parasitaemia and gametocytaemia.

PQ is used as a causal prophylactic agent not a blood schizonticidal agent due to relatively greater activity against liver schizonts than blood schizonts [28-31] and in this $P$. berghei murine model, $20 \mathrm{mg} / \mathrm{kg}$ PQ causally protected all mice but led to recrudescence when used as a treatment for erythrocytic infection. It may be suggested that this comparability to clinical experience enhances the value of the $P$. berghei murine model and that it may be useful in screening new 8-aminoquinolines and novel drug classes in causal prophylactic and erythrocyte treatment studies.

The somewhat lower prophylactic efficacy of PQ compared to TQ after multiple administrations of both drugs has also been observed clinically. In a review, the efficacy of PQ (approximately $0.5 \mathrm{mg} / \mathrm{kg} /$ day from the day prior to parasite exposure until 1 week after parasite exposure) for $P$. falciparum ranged from 85 to $95 \%$ in five studies. 

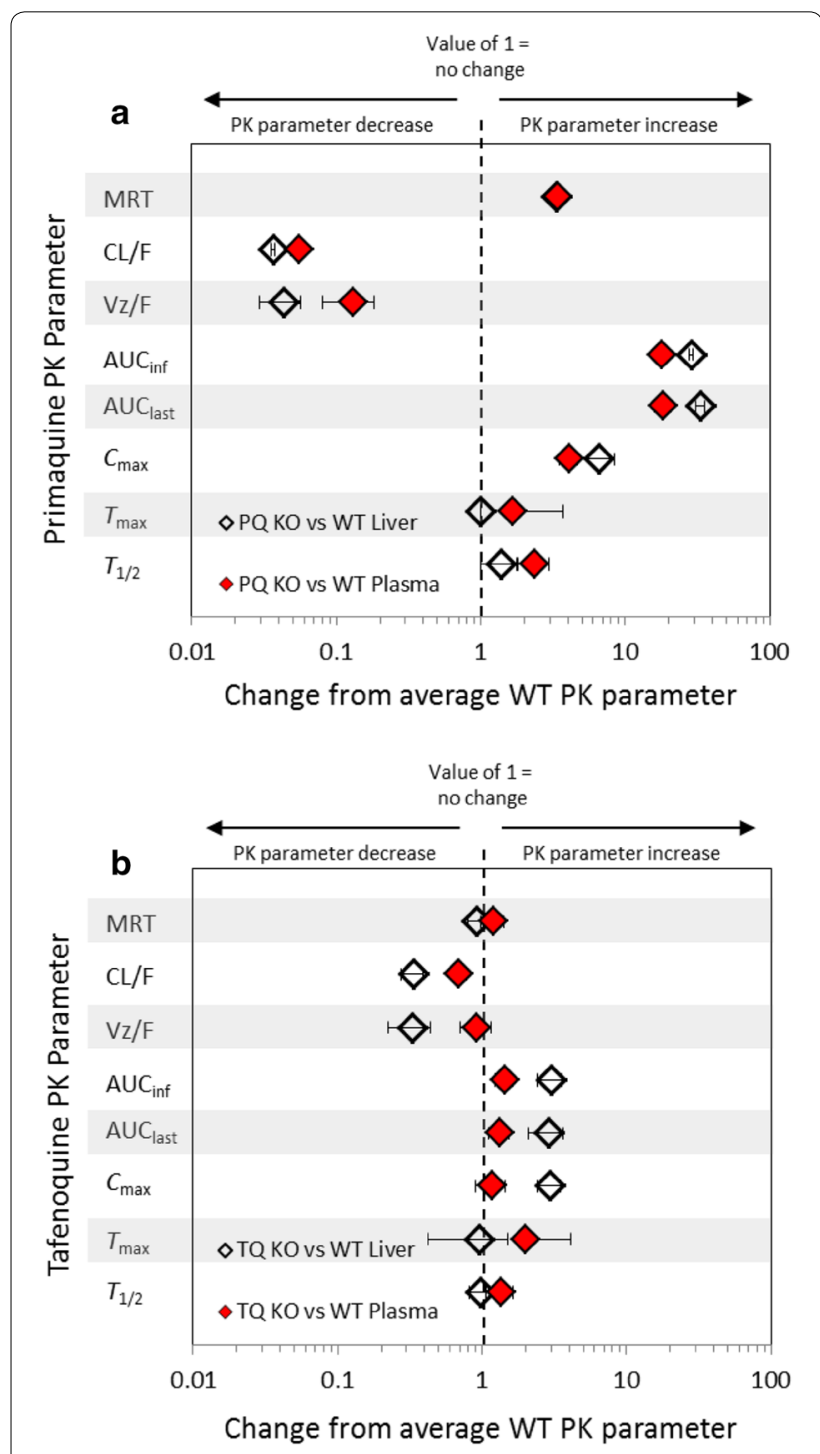

Fig. 3 Relative fold changes of primaquine and tafenoquine CYP2D KO pharmacokinetic parameters from reference (WT parameters) in mouse plasma and liver. Indicated are the fold changes after the administration $20 \mathrm{mg} / \mathrm{kg}$ primaquine $(P Q)$ and $20 \mathrm{mg} / \mathrm{kg}$ tafenoquine (TQ) in the CYP2D KO strain as compared to WT C57BL/6 mice. The fold change is indicated by the $x$-axis and the pharmacokinetic parameter on the $y$-axis. A value of one represents no relative change in the pharmacokinetic parameter between the KO and WT mice. The grey bars are provided for visual reference. The error shown is the standard deviation of relative fold changes for $\mathrm{KO}$ pharmacokinetic parameters as compared to WT means pharmacokinetic parameter values. PQ and TQ comparisons were conducted using experimental data generated at the WRAIR [26, 27]. WT C57BL/6 wild-type mice. CYP2D KO C57BL CYP2D6 knock-out mice. MRT mean resonance time. CL/F apparent total clearance of the drug from plasma after oral administration. Vz/F apparent volume of distribution during terminal phase after nonintravenous administration. AUCinf area under the plasma concentration-time curve from time zero to infinity. AUClast area under the plasma concentration-time curve from time zero to the time of last measurable concentration. Cmax: maximum (peak) plasma drug concentration. Tmax time to reachmaximum (peak) plasma concentration following drug administration. $T 1 / 2$ elimination half-life
[1] In contrast, the efficacy of TQ $(200 \mathrm{mg} /$ day for 3 days as loading dose followed by $200 \mathrm{mg}$ weekly) was $100 \%$ in non-immune Australian troops in comparison to mefloquine efficacy of $100 \%$ and placebo failure rate estimated at $8 \%$ [32]. TQ efficacy was $98 \%$ in semi-immune Africans where the efficacy of mefloquine was also $98 \%$ and the placebo failure rate was $32 \%$ [33]. This PQ versus TQ comparison supports the PQ hepatic versus erythrocytic infection comparison of the prior paragraph and again suggests that the $P$ berghei mouse model may be reasonably comparable to the clinical situation.

If the $P$. berghei model has clinical relevance, our work suggests that CYP2D6 polymorphisms will not be important for TQ erythrocytic clinical activity. The lack of effect due to CYP2D-cluster deletion on TQ erythrocytic activity is in contrast to the previous demonstration of CYP2D-mediated activity relative to liver schizonts in mice $[11,13]$. Since TQ prophylactic activity is thought to require some efficacy against blood-stage asexual parasites, the present work suggests that prospective human prophylactic candidates do not need to be screened for 2D6 polymorphisms.

Given that CYP2D-mediated metabolism is required for causal prophylaxis and radical cure (in the case of primaquine) but not for the eradication of the erythrocytic parasites, it is reasonable to suppose that 8-aminoquinolines exhibit different modes of action against different stages of the parasite. Clearing parasites from hepatocytes may involve metabolic activation to oxidative intermediates [11-13], which are produced at lower concentrations in CYP2D KO mice relative to WT mice $[26,27]$. In the case of erythrocytic stages, inhibition of haematin agglutination has been proposed [34], although this leads to further questions regarding the mechanistic rational related to the pluripotentiality of 8-aminoquinolines and warrants further exploration.

\section{Conclusion}

Overall, this C57BL murine model mimics the clinical experience for PQ and TQ and may be useful in screening new 8-aminoquinolines and potentially novel drug classes to directly compare causal prophylactic and treatment dosing regimens. The model allowed for the direct comparison of causal prophylaxis and treatment modalities and indicates that PQ inhibits hepatic infections at lower doses than were required to treat erythrocytic infections. In contrast, TQ exhibits analogous dose-response relationships relative to hepatic and erythrocytic infections in wild-type animals. In addition, TQ clears erythrocytic infections at lower doses than PQ. The data comparing wild-type and geneticallymodified mice suggest that single-dose administration of TQ and PQ are not impacted by CYP2D mediated 
metabolism relative to the initial clearing of erythrocytic $P$. berghei infections to the same extent as hepatic infections.

\section{Additional file}

Additional file 1. Figure S1. Primaquine causal prophylactic (hepatic) IVIS activity (refer to manuscript Table 2). Figure S2 (Part $1 / 2$ and Part 2/2): Tafenoquine causal prophylactic (hepatic) IVIS activity (refer to manuscript Table 3). Figure S3. Comparison of IVIS vehicle control wild-type (WT) and CYP2D knock-out (KO) C57BL mice. Figure S4. Individual IVIS bioluminescence signal values measured in C57BL/6 WT and CYP2D KO mice. Figure S5. Forrest Plot of percent parasitaemia following $40 \mathrm{mpk}$ PQ or $25 \mathrm{mpk} T \mathrm{Q}$ administered day 4 post IV sporozoite challenge (day 0). Figure S6. Forrest Plot of percent gametocytaemia (mature gametocytes) following $40 \mathrm{mpk}$ PQ or $25 \mathrm{mpk}$ TQ administered day 4 post IV sporozoite challenge. Figure S7. Correlation between flow cytometry and light microscopy (based upon percent parasitaemia). Solid line represents linear regression. Figure S8. Genetic verification of a subset of C57BL wild-type and CYP2D knock-out mice.

\section{Authors' contributions}

EEM: drafted the manuscript, developed project plan, served as project manager, prepared and read microscopy slides, and performed data analyses. JB: provided mentorship and guidance during EEM's clinical pharmacology fellowship. DC: generated the In Vivo Imaging System (IVIS) data. SPD: performed the statistical analysis. MRH: serves as the Division Director of Experimental Therapeutics at the WRAIR and provided guidance and mentorship during EEM's clinical pharmacology fellowship. PJL, BAV, and NR: quantified parasitemia and gametocytemia via microscopy (NR also dissected mosquitos and generated sporozoites). LTR: serves as the Program Manager and Chair of the Tafenoquine Integrated Project Team. SRM: contributed to the generation of Fig. 3. LHX, JZ, PZ: executed the animal care/handling/monitoring/euthanasia, IV sporozoite challenge, drug dosing, blood sampling, and flow cytometry work. QL: served as Chief of the In Vivo Section and principle investigator of the animal protocol. All authors reviewed the manuscript prior to submission.

\section{Author details}

${ }^{1}$ Walter Reed Army Institute of Research (WRAIR), Silver Spring, MD, USA.

${ }^{2}$ United States Army Medical Materiel Development Authority (USAMMDA), Ft Detrick, Frederick, MD, USA.

\section{Acknowledgements}

This material has been reviewed by the Walter Reed Army Institute of Research. There is no objection to its presentation and/or publication. The opinions or assertions contained herein are the private views of the authors and are not to be construed as official or as reflecting true views of the Department of the Army or the Department of Defense. All animal experiments were conducted in a facility accredited by the Association for the Assessment and Accreditation of Laboratory Animal Care, International, and in compliance with the Animal Welfare Act and other federal statutes and regulations relating to animals and experiments involving animals and adhere to the principles stated in the Guide for the Care and Use of Laboratory Animals (National Academy Press, 1996).

\section{Competing interests}

The authors declare that they have no competing interests.

\section{Availability of data and material}

The datasets during and/or analysed during the current study available from the corresponding author on reasonable request.

\section{Funding}

This work was supported by the Military Infectious Diseases Research Program (MIDRP), project number Q0433_15WR_CS and Q0435_15_WR_CS and Defense Health Program (DHP) P8 funds.
Received: 17 August 2016 Accepted: 22 November 2016

Published online: 07 December 2016

\section{References}

1. Hill DR, Baird JK, Parise ME, Lewis LS, Ryan ET, Magill AJ. Primaquine: report from CDC expert meeting on malaria chemoprophylaxis I. Am J Trop Med Hyg. 2006;75:402-15.

2. FDA. Primaquine Phosphate Label. 2015. http://www.accessdata.fda.gov/ drugsatfda_docs/label/2015/008316s021 lbl.pdf. Accessed 23 Dec 2015.

3. Baird JK. Drug therapy: effectiveness of antimalarial drugs. N Engl J Med. 2005;352(15):1565-77.

4. WHO. Updated WHO policy recommendation: single dose primaquine as a gametocytocide in Plasmodium falciparum malaria. Geneva, World Health Organization, 2012. http://www.who.int/malaria/publications/ atoz/who_pq_policy_recommendation/en/. Accessed 30 Dec 2015.

5. Rajapakse S, Rodrigo C, Fernando SD. Tafenoquine for preventing relapse in people with Plasmodium vivax malaria. Cochrane Database Syst Rev. 2015:4:10458

6. Crockett M, Kain KC. Tafenoquine: a promising new antimalarial agent. Expert Opin Investig Drugs. 2007;16:705-15.

7. White NJ, Qiao LG, Qi G, Luzzatto L. Rationale for recommending a lower dose of primaquine as a Plasmodium falciparum gametocytocide in populations where G6PD deficiency is common. Malar J. 2012;11:418.

8. Baird JK. Primaquine toxicity forestalls effective therapeutic management of the endemic malarias. Int J Parasitol. 2012;42:1049-54.

9. Vale N, Moreira R, Gomes P. Primaquine revisited six decades after its discovery. Eur J Med Chem. 2009;44:937-53.

10. Bennett JW, Pybus BS, Yadava A, Tosh D, Sousa JC, McCarthy WF, et al. Primaquine failure and cytochrome P-450 2D6 in Plasmodium vivax malaria. N Engl J Med. 2013;369:1381-2.

11. Pybus BS, Marcsisin SR, Jin X, Deye G, Sousa JC, Li Q, et al. The metabolism of primaquine to its active metabolite is dependent on CYP 2D6. Malar J. 2013;12:212.

12. Marcsisin SR, Reichard G, Pybus BS. Primaquine pharmacology in the context of CYP 2D6 pharmacogenomics: current state of the art. Pharmacol Ther. 2016;161:1-10.

13. Marcsisin SR, Sousa JC, Reichard GA, Caridha D, Zeng Q, Roncal N, et al. Tafenoquine and NPC-1161B require CYP 2D metabolism for anti-malarial activity: implications for the 8-aminoquinoline class of anti-malarial compounds. Malar J. 2014;13:2.

14. Deye GA, Magill AJ. Primaquine for prophylaxis of malaria: has the CYP sailed? J Travel Med. 2014;21:67-9.

15. Bradford LD. CYP2D6 allele frequency in European Caucasians, Asians, Africans and their descendants. Pharmacogenomics. 2002;3:229-43.

16. Milner E, Sousa J, Pybus B, Auschwitz J, Caridha D, Gardner S, et al. Ketotifen is an antimalarial prodrug of norketotifen with blood schizonticidal and liver-stage efficacy. Eur J Drug Metabol Pharmacokinet. 2012;37:17-22.

17. Li QG, O'Neil M, Xie L, Caridha D, Zeng Q, Zhang J, et al. Assessment of the prophylactic activity and pharmacokinetic profile of oral tafenoquine compared to primaquine for inhibition of liver stage malaria infections. Malar J. 2014;13:141.

18. Scheer N, Kapelyukh Y, McEwan J, Beuger V, Stanley LA, Rode A, et al. Modeling human cytochrome P450 2D6 metabolism and drug-drug interaction by a novel panel of knockout and humanized mouse lines. Mol Pharmacol. 2012;81:63-72.

19. Allard STM, Kopish K. Luciferase reporter assays: powerful, adaptable tools for cell biology research. Cell Notes. 2008;21:23-6.

20. Ozaki LS, Gwadz RW, Godson GN. Simple centrifugation method for rapid separation of sporozoites from mosquitoes. J Parasitol. 1984;70:831-3.

21. Xie L, Li Q, Johnson J, Zhang J, Milhous W, Kyle D. Development and validation of flow cytometric measurement for parasitaemia using autofluorescence and YOYO-1 in rodent malaria. Parasitology. 2007;134:1151-62.

22. Li QG, Gerena L, Xie L, Zhang J, Kyle D, Milhous W. Development and validation of flow cytometric measurement for parasitemia in cultures of P. falciparum vitally stained with YOYO-1. Cytometry A. 2007;71:297-307. 
23. Thurston JP. The morphology of Plasmodium berghei before and after treatment with drugs. Trans R Soc Trop Med Hyg. 1953;47:248-56.

24. Kreier JP, Mohan R, Seed T, Pfister RM. Studies of morphology and survival characteristics of erythrocytes from mice and rats with Plasmodium berghei infection. Z Tropenmed Parasitol. 1972;23:245-55.

25. Centrum LUM. 2015. https://www.lumc.nl/org/parasitologie/research/ malaria/berghei-model/Morphology-berghei-LM/Morphology-bergheiLM-images/. Accessed 18 Dec 2015.

26. Potter BM, Xie LH, Vuong C, Zhang J, Zhang P, Duan D, et al. Differential CYP 2D6 metabolism alters primaquine pharmacokinetics. Antimicrob Agents Chemother. 2015;59:2380-7.

27. Vuong C, Xie LH, Potter BM, Zhang J, Zhang P, Duan D, et al. Differential cytochrome P450 2D metabolism alters tafenoquine pharmacokinetics. Antimicrob Agents Chemother. 2015;59:3864-9.

28. St Jean PL, Xue Z, Carter N, Koh GC, Duparc S, Taylor M, et al. Tafenoquine treatment of Plasmodium vivax malaria: suggestive evidence that CYP2D6 reduced metabolism is not associated with relapse in the Phase $2 \mathrm{~b}$ DETECTIVE trial. Malar J. 2016:15:97.

29. Arnold J, Alving AS, Hockwald RS, Clayman CB, Dern RJ, Beutler E, et al. The effect of continuous and intermittent primaquine therapy on the relapse rate of Chesson strain vivax malaria. J Lab Clin Med. 1954:44:429-38.
30. Baird JK, Wiady I, Sutanihardja A, Suradi Purnomo, Basri H, et al. Therapeutic efficacy of chloroquine combined with primaquine against Plasmodium falciparum in northeastern Papua Indonesia. Am J Trop Med Hyg. 2002;66:659-60.

31. Pukrittayakamee S, Vanijanonta S, Chantra A, Clemens R, White NJ. Blood stage antimalarial efficacy of primaquine in Plasmodium vivax malaria. J Infect Dis. 1994;169:932-5.

32. Dow GS, McCarthy WF, Reid M, Smith B, Tang D, Shanks GD. A retrospective analysis of the protective efficacy of tafenoquine and mefloquine as prophylactic anti-malarials in non-immune individuals during deployment to a malaria-endemic area. Malar J. 2014;13:49.

33. Dow GS, Liu J, Lin G, Hetzell B, Thieling S, McCarthy WF, et al. Summary of anti-malarial prophylactic efficacy of tafenoquine from three placebocontrolled studies of residents of malaria-endemic countries. Malar J. 2015;14:473.

34. Vennerstrom JL, Nuzum EO, Miller RE, Dorn A, Gerena L, Dande PA, et al. 8-aminoquinolines active against blood stage Plasmodium falciparum in vitro inhibit hematin polymerization. Antimicrob Agents Chemother. 1999;43:598-602.

\section{Submit your next manuscript to BioMed Central and we will help you at every step:}

- We accept pre-submission inquiries

- Our selector tool helps you to find the most relevant journal

- We provide round the clock customer support

- Convenient online submission

- Thorough peer review

- Inclusion in PubMed and all major indexing services

- Maximum visibility for your research

Submit your manuscript at www.biomedcentral.com/submit
() Biomed Central 